\title{
A Novel Machine Learning-Based Radiomic Model for Diagnosing High Bleeding Risk Esophageal Varices in Cirrhotic Patients
}

\section{Yijie Yan}

Capital Medical University Youan Hospital

\section{Yue Li}

Fourth Affiliated Hospital of Harbin Medical University

\section{Chunlei Fan}

Capital Medical University Youan Hospital

\section{Yuening Zhang}

Capital Medical University Youan Hospital

\section{Shibin Zhang}

Capital Medical University Youan Hospital

\section{Zhi Wang}

Blot info and tech Co.Itd

\section{Tehui Huang}

Blot info and Tech Co.Ltd

\section{Zhenjia Ding}

Chinese Academy of Sciences

\section{Keqin $\mathrm{Hu}$}

University of California Irvine

Lei Li

Capital Medical University Youan Hospital

Huiguo Ding ( $\nabla$ dinghuiguo@medmail.com.cn )

Beijing Youan Hospital https://orcid.org/0000-0002-8716-4926

\section{Research Article}

Keywords: Development and validation, Cirrhosis, Computed tomography, Portal hypertension, Al, 5-fold cross validation, SVM, Non-invasive, AUROC, Endoscopy

Posted Date: September 21st, 2021

DOl: https://doi.org/10.21203/rs.3.rs-889812/v1 
License: (c) (i) This work is licensed under a Creative Commons Attribution 4.0 International License. Read Full License

Version of Record: A version of this preprint was published at Hepatology International on April 2nd, 2022. See the published version at https://doi.org/10.1007/s12072-021-10292-6. 


\section{Abstract}

Background \& aims: To develop and validate a novel machine learning-based radiomic model (RM) for diagnosing high bleeding risk esophageal varices (HREV) in cirrhosis.

Methods: In training cohort, total 218 cirrhotic patients for mild esophageal varices (EV) and 240 for HREV RM were enrolled for training and internal validation. In external validation cohort, 159 and 340 cirrhotic patients were respectively used for mild EV and HREV RM validation. Interesting regions of liver, spleen, and esophagus were labeled on the portal venous-phase enhanced CT images. RM was assessed by area under the receiver operating characteristic curves (AUROC), sensitivity, specificity, calibration and decision curve analysis (DCA).

Results: The AUROC of mild EV RM in training and internal validation was 0.943 and 0.732 , sensitivity and specificity was $0.863,0.773$ and $0.763,0.763$. The AUROC, sensitivity and specificity was 0.654 , 0.773 and 0.632 in external validation. Interestingly, the AUROC of HREV RM in training and internal validation was 0.983 and 0.834 , sensitivity and specificity was $0.948,0.916$ and $0.977,0.969$. The AUROC, sensitivity and specificity was $0.736,0.690$ and 0.762 in external validation. Calibration and DCA indicated RM had good performance in clinical practice. Compared with Baveno VI and its expanded criteria, HREV RM had a higher accuracy and net reclassification improvement reached $49.0 \%$ and $32.8 \%$.

Conclusion: A novel non-invasive RM for diagnosing HREV in cirrhotic patients with highly accuracy was developed. However, this RM still needs to be validated by a multi-center large cohort.

\section{Introduction}

The esophageal varices(EV) bleeding(EVB) is one of the lethal complications of liver cirrhosis with portal hypertension. The 6-week mortality of acute EVB reaches more than 15\%[1].The 3- and 5-year cumulative mortality are $24.7 \%$ and $47.2 \%$ in cirrhotic patients by endoscopic or/and medicine therapy for secondary prophylaxis after first episode[2]. Therefore, it is very important to diagnosing EV in cirrhotic patients, especially moderate-severe EV, namely high bleeding risk esophageal varices(HREV) needed treatment. Currently, the endoscopy is still "gold standard" for evaluation degree of EV. Guidelines recommend that all patients should undergo endoscopy once cirrhosis is confirmed in patients with chronic liver diseases and surveillance EV every from 1 to 3 years $[3,4]$. However, endoscopy is an invasive procedure and may bring complications such as bleeding, cardiac tear, and discomfort. Hepatic vein pressure gradient (HVPG), as an alternative indicator of portal pressure, indicates an increased bleeding risk of EV when HVPG is more than $12 \mathrm{mmHg}$. However, HVPG measurement is not only an invasive operation but also requires skilled operators to perform[5]. Thus, it is important to develop a non-invasive method to screen EV and diagnose HREV to avoid unnecessary endoscopy [6, 7]. Based on the meta-analysis of observational studies recently, it suggested that computed tomography (CT) imaging, a non-invasive diagnostic tool, is a good choice for evaluation of EV in cirrhotic patients[8]. However, contrast-enhanced CT images have a large amount of features that cannot be completely distinguished and interpreted by 
doctors. Through artificial intelligence (Al) technology, namely machine learning algorithm, we can analyze unordered and underlying pathophysiological features[9]. Therefore, the aim of this study is to develop and validate a novel radiomic model (RM) for diagnosing HREV in cirrhotic patients based on machine learning algorithm with contrast-enhanced CT images.

\section{Methods}

\section{Study design}

This is a retrospective single center multi-cohort study. The inclusion criteria were: (1) age18-85 years old, (2) patients diagnosed with cirrhosis, confirmed by histopathology and/or typical symptoms and signs of laboratory and imaging findings, including abdominal ultrasonography[10,11], (3) patients who underwent endoscopy and contrast-enhanced CT scan, the interval time between CT scan and endoscopy should be within 3 months. The exclusion criteria were: (1) patients with non-cirrhotic portal hypertension, (2) patients who previously underwent one of the following therapies: transjugular intrahepatic portosystemic shunt, splenectomy, partial splenic embolization, or endoscopic variceal therapy (endoscopic varicel ligation and injection sclerotherapy), (3) patients diagnosed with primary or metastatic hepatocellular carcinoma or other tumors, (4) pregnant or lactation patients. The study protocol was performed in compliance with the Declaration of Helsinki and approved by the Ethics Committees of Beijing You'an Hospital Capital Medical University (Approved number: 2019-074). Signed informed consent was obtained from each patient for using samples, materials and publication.

\section{Subjects}

Total 796 qualified participants were enrolled for study. In training cohort, 391 patients with cirrhosis due to any cause were enrolled between January 2016 to December 2017 for. Among them, patients with mild EV (mild EV, n=120), moderate-severe EV (HREV, n=162) and without EV (non EV, $n=109)$ were included. 109 patients were randomly selected from mild EV group to match patients without EV, 120 patients were randomly selected from HREV group to match patients with mild EV to balance the number of cases. Ultimately, 218 (non EV and mild EV) and 240 (mild EV and HREV) patients were separated into the training and internal validation of mild EV and HREV RM (Figure 1a). Additionally, in external validation cohort, 405 cirrhotic patients with hepatitis B from January 2018 to December 2019 were consecutively enrolled, including mild EV( $n=94)$ or HREV $(n=246)$ or non EV $(n=65) .159$ (non EV and mild EV) and 340 (mild EV and HREV) patients were respectively used for external validation of mild EV and HREV RM (Figure 1a).

\section{Clinical data and Biochemistry}

Clinical data included sex, age, laboratory test results, diagnosis, underlying etiology, and complications of cirrhosis/portal hypertension from the electronic medical record. The etiologies of viral cirrhosis were hepatitis $\mathrm{B}$ (serum HBsAg positive), hepatitis $\mathrm{C}$ (serum anti-HCV and HCV-RNA positive). Cirrhosis due to alcoholic, autoimmune and metabolism-related fatty liver diseases were identified according to 
guideline[12,13,14]. Platelets counts (PLT) were analyzed with XE-5000 (Sysmex, Kobe, Japan). International normalized ratio (INR) and prothrombin time (PT) were measured by an automatic coagulation analyzer (ACLTOP 700, Instrumentation Laboratory Company, USA). An automatic biochemical analyzer (AU5400, Olympus Company, Tokyo, Japan) was used to measure biochemistry of liver and renal, including serum aspartate aminotransferase (AST), alanine aminotransferase (ALT), total bilirubin (TB), albumin (ALB), and creatinine (Cr).

\section{EV evaluation by endoscopy}

The flexible GI endoscope (GIF-CV290 or GIF-CV260, Olympus, Japan) was used. The endoscopy was carried out by the experienced gastrointestinal physician and reports were consistently reviewed by two hepatologists to classify the degree of EV as follows[15]: mild EV: linear or slightly tortuous $\mathrm{EV}(\leq 3 \mathrm{~mm}$ diameter) without red signs (Figure 2a); moderate EV: mild EV with red signs or EV with snake or tortuous bulges (3-10 mm diameter) without red signs (Figure 2b); severe EV: medium EV with red signs, or beadlike, or nodular form or EV $(>10 \mathrm{~mm})$, regardless of whether there is a red sign or not (Figure $2 \mathrm{c})$. The moderate-severe EV was regarded as HREV in need of treatment[16].

\section{Contrast-enhanced CT image acquisition}

All enrolled patients had abdominal contrast-enhanced abdominal CT using 64-slice CT scanner (GE Lightspeed VCT, GE Healthcare, USA). The following parameters were used-collimation, $0.625 \mathrm{~mm}$; tube voltage, $120 \mathrm{kVp}$; tube current, $380 \mathrm{~mA}$; slice thickness, $5 \mathrm{~mm}$; pitch, 1.375. All patients received an intravenous, lopromide (iodine concentration, $370 \mathrm{mg} / \mathrm{mL}$ ) $100 \mathrm{ml}$ via an antecubital vein at a rate of 3 $\mathrm{ml} / \mathrm{s}$ using a high-pressure injector. All the patients underwent triple-phase contrast-enhanced scans.

\section{Radiomic features extraction}

All radiomic data and development of RM were programmed in the Python 3.5 based on Ubuntu 16.04.4. Firstly, Regions of interest (ROI) were created manually on the portal vein phase of contrastenhanced CT images (DICOM data). Two hepatologists carefully identified tissues in application(AIMED, Version 2.2.3, Beijing Blot Info \& Tech Co. Ltd, China) on each slice and labeled liver, spleen and esophagus as ROI (Figure 2d). Since the lesion parts of EV mostly occured within $5 \mathrm{~cm}$ esophagus above the cardia[17], ROI of esophagus were labeled in this area. If there was a different opinion on choosing ROI, it will be decided after the two hepatologists review together. 3D reconstruction with marching cubes algorithm and radiomic features extraction were performed on the marked ROI areas reconstructed as $1 \times 1 \times 1 \mathrm{~mm}$ voxel. The above reconstruction process could reduce the difference of image pixel spacing and form 3D ROI. Four groups of radiomic features were extracted from the segmented ROI including textural features, statistical features, wavelet features and histogram of oriented gradient (HOG) features. Finally, the evaluator were blinded on the patient's endoscopic results in advance.

\section{Dimension reduction}


The extraction of imaging features produced a lot of data, which will reduce the efficiency of models development. To simplify the data analysis, we used principal components analysis (PCA) to make dimension reduction. The process is as following. First, invalid image omics features (such as infinite value, null value, features with variance of 0 , etc.) are removed, the remaining features are standardized and a covariance matrix was created to obtain the eigenvalues and eigenvectors; Equation is as follows: $\mathrm{Xi}^{\prime}=(\mathrm{Xi}-\mathrm{Xmin}) /(\mathrm{Xmax}-\mathrm{Xmin}) . \mathrm{Xi}^{\prime}$ : The image features of the patient standardized in this dimension. Xi: The image features of the patient non-standardized in this dimension. Xmax: The maximum value of this dimension. Xmin: The minimum value of this dimension. Then, sorting the eigenvalues from large to small, selecting the principal components, finding their corresponding eigenvectors, projecting the original dimension data onto the selected eigenvectors, the dimensions of the original data characteristics were reduced, and applying the selected dimension data to represent the original data for processing and analysis .

\section{Machine learning algorithm and development of RM}

We tested several classification algorithms diagnosing of mild EV or HREV in training cohort. Finally, support vector machine (SVM) with highest AUC and ACC was selected to establish the model. The basic purpose of SVM is to find the best separating hyperplane on the feature space so that the interval between positive and negative samples in the training cohort is maximized. The aim of this algorithm is to minimize the function value:

$L(\omega, b, \alpha)=\frac{1}{2}\|\omega\|^{2}-\sum_{i=1}^{n} \alpha_{i}\left[y_{i}\left(\omega^{T} x_{i}+b\right)-1\right]$

$\frac{1}{2}\|\omega\|^{2}$ $\left(\sum_{\mathrm{i}=1}^{\mathrm{n}} \alpha_{\mathrm{i}}\left[\mathrm{y}_{\mathrm{i}}\left(\omega^{\mathrm{T}} \mathrm{x}_{\mathrm{i}}+\mathrm{b}\right)-1\right]\right)$, cross-validation were applied to evaluate models and optimize parameters Figure $1 \mathrm{~b}$. The participants were randomly divided into 5 parts, one part was used as training and development of RM, and the other 4 parts was used as internal validation. Five models could be obtained and efficiency evaluation was reflected in the form of average value. The RM with minimum mean squared error (MSE) in 5-fold internal cross-validation was selected as the optimization. After retraining optimal RM with all participants, the final RM was obtained which used for external validation.

\section{Efficacy evaluation of RM}

AUROC, sensitivity, specificity, accuracy were calculated as indicators to evaluate the discrimination of RM. Calibration curve reflects the agreement between predictions from the models and observed outcomes, and decision curve analysis (DCA) was applied to determine the clinical usefulness of the RM net benefits at different threshold probabilities. Baveno VI consensus proposed that cirrhotic patients with 
LSM $<20 \mathrm{kpa}$ and PLT $>150 * 10^{9} / \mathrm{L}$ had low EVB risks and could avoid endoscope tests. ${ }^{16}$ The expanded Baveno VI criteria extended the threshold to LSM $<25 \mathrm{kpa}$ and PLT $>110 * 10^{9} / \mathrm{L}[18]$. In other words, people who exceed these criteria are at risk of EV bleeding and should be screened with gastroscopy. We would evaluate the efficacy of RM diagnosing HREV by accuracy and net reclassification improvement (NRI) in external validation cohort compared with Baveno VI, expanded Baveno VI criteria respectively.

\section{Statistical analysis}

Statistical Package for Social Sciences (SPSS, version 26.0, IBM Corp.; Armonk, NY, USA) was used for analysis data. The mean and standard deviation(SD) were expressed for normally distributed quantitative data. The median and inter-quartile range were expressed in non-normally distributed data. Normally distributed data matrixes were compared by the independent Student's t test, while non-normally distributed data matrixes were compared by Mann-Whitney $U$ test. Frequencies and proportions were used to summarize enumeration data with Chi-square test. All authors had access to the study data and reviewed and approved the final manuscript.

\section{Results}

\section{Clinical characteristics}

The flow chart of qualified participants were shown in Fig. 1a-b. The clinical characteristics of enrolled patients in training and external validation cohort were summarized in Table 1 in detail. 
Table 1

The clinical characteristics in training and external validation cohort

\begin{tabular}{|c|c|c|c|c|c|c|}
\hline & Training coh & & External vali & on cohort & $P^{\#}$ & $P^{*}$ \\
\hline & $\begin{array}{l}\text { Mild EV } \\
\text { model } \\
(n=218)\end{array}$ & $\begin{array}{l}\text { HREV } \\
\text { model } \\
(n=240)\end{array}$ & $\begin{array}{l}\text { Mild EV } \\
\text { model } \\
(n=159)\end{array}$ & $\begin{array}{l}\text { HREV } \\
\text { model } \\
(n=340)\end{array}$ & & \\
\hline Age(year) ${ }^{\wedge}$ & $53.6 \pm 10.5$ & $54.9 \pm 10.5$ & $52.2 \pm 10.6$ & $53.9 \pm 10.7$ & .204 & .264 \\
\hline $\begin{array}{l}\text { Sex } \\
\text { Male } \\
\text { Female }\end{array}$ & $\begin{array}{l}138(63.3) \\
80(36.7)\end{array}$ & $\begin{array}{l}158(65.8) \\
82(34.2)\end{array}$ & $\begin{array}{l}94(59.1) \\
65(40.9)\end{array}$ & $\begin{array}{l}224(65.9) \\
116(34.1)\end{array}$ & .410 & .990 \\
\hline Etiology $\$$ & $110(50.5)$ & 104(43.3) & 159(100.0) & $340(100.0)$ & & \\
\hline HBV & 40(18.3) & $54(24.0)$ & 0 & 0 & & \\
\hline Alcohol & $10(4.6)$ & $18(7.5)$ & 0 & 0 & & \\
\hline $\mathrm{HCV}$ & $6(2.8)$ & $0(0)$ & 0 & 0 & & \\
\hline $\mathrm{HBV}+\mathrm{HCV}$ & $20(9.2)$ & $18(7.5)$ & 0 & 0 & & \\
\hline Atutoimmune & $2(0.9)$ & $4(1.7)$ & 0 & 0 & & \\
\hline $\begin{array}{l}\text { NASH } \\
\text { Other }\end{array}$ & $30(13.7)$ & $42(17.5)$ & 0 & 0 & & \\
\hline $\operatorname{ALT}(\mathrm{U} / \mathrm{L})^{\&}$ & $\begin{array}{l}25.6 \\
(17.0-44.1)\end{array}$ & $\begin{array}{l}23.2 \\
(16.0-37.0)\end{array}$ & $\begin{array}{l}26.0 \\
(16.0-39.0)\end{array}$ & $\begin{array}{l}25.0 \\
(18.0-34.0)\end{array}$ & .543 & .385 \\
\hline AST(U/L)\& & $\begin{array}{l}39.0 \\
(25.0-59.4)\end{array}$ & $\begin{array}{l}37.0 \\
(27.0-56.8)\end{array}$ & $\begin{array}{l}32.0 \\
(23.0-44.0)\end{array}$ & $\begin{array}{l}32.5 \\
(25.0-45.0)\end{array}$ & .003 & .001 \\
\hline TB(umol/L)\& & $\begin{array}{l}24.4 \\
(15.5-49.5)\end{array}$ & $\begin{array}{l}31.0 \\
(18.4-55.9)\end{array}$ & $\begin{array}{l}21.3 \\
(14.1-28.2)\end{array}$ & $\begin{array}{l}24.1 \\
(17.1-35.1)\end{array}$ & .002 & .001 \\
\hline $\operatorname{ALB}(g / L)^{\wedge}$ & $35.4 \pm 6.5$ & $33.3 \pm 5.6$ & $36.8 \pm 6.8$ & $36.2 \pm 6.4$ & .045 & .001 \\
\hline
\end{tabular}

\# Indicates the comparison between mild model in training and external validation cohort, * Indicates the comparison between HREV model in training and external validation cohort, ${ }^{\wedge}$ Data are presented as mean \pm standard deviation, \& Data are presented as median(Interquartile distance), \$ Data are presented as $\mathrm{n}(\%)$.

Abbreviation HBV Hepatitis B virus, HCV Hepatitis C virus, NASH Nonalcoholic steatohepatitis, ALT alanine aminotransferase, AST aspartate aminotransferase, TB total bilirubin, ALB albumin, $\mathrm{Cr}$ creatinine, PLT platelet count, INR international normalized ratio 


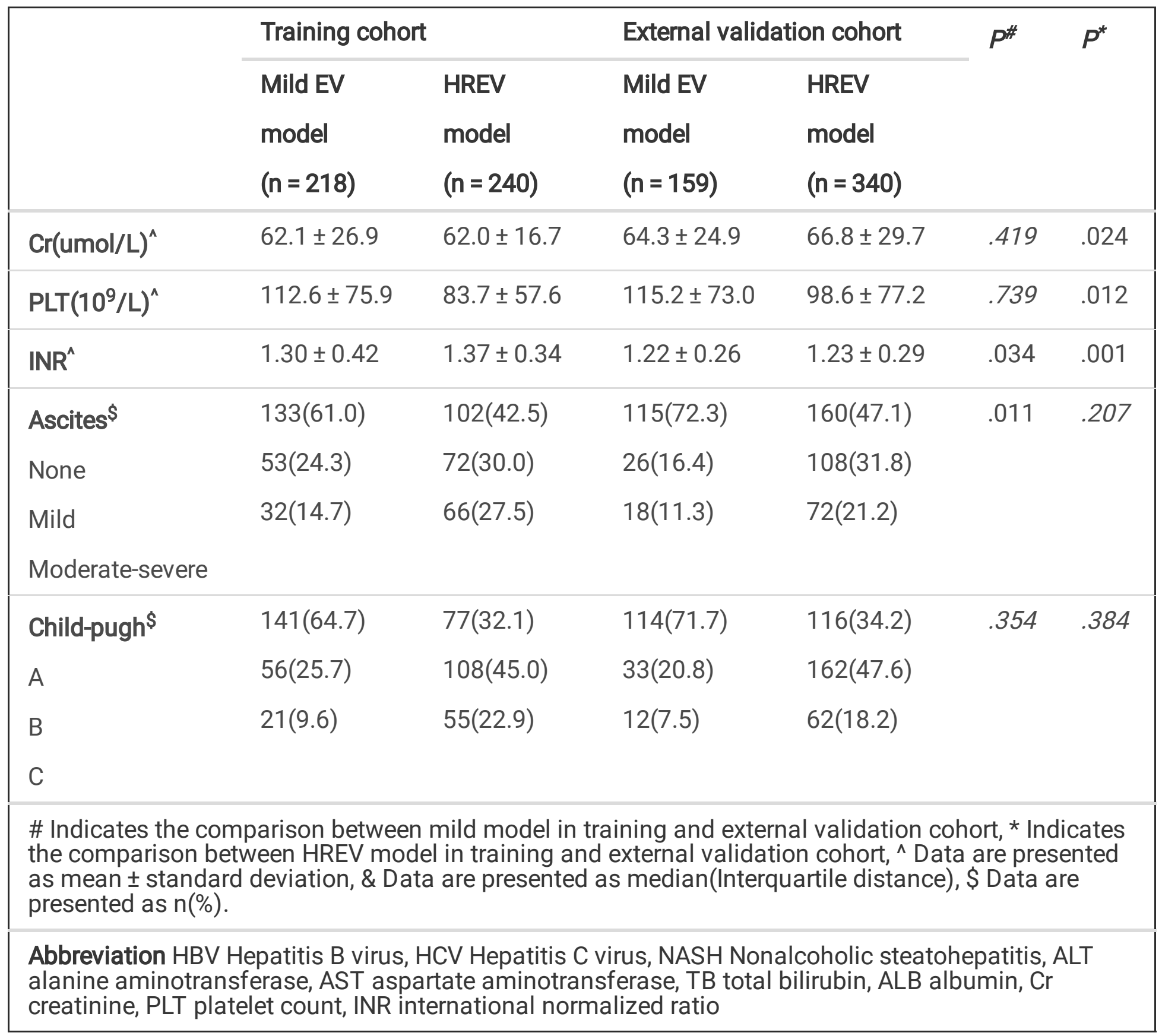

\section{Radiomic Feature And Development Of Rm}

Finally, it extracted total 2358 imaging features in 3 ROIs, including 42 textural features, 156 statistical features, 48 wavelet features and 540 HOG features in each ROI. After dimension reduction through PCA, the RM for mild EV and HREV contained 163 and 177 features, respectively. During RM training, the best penalty parameter $(C=0.85)$ which enable SVM algorithm optimized was determined by 5 -fold crossvalidation. In order to find best kernel function, we went through all the possible kernel functions.

\section{Evaluation Of Rm}


The average AUROC of RM for mild EV in the training was 0.943 (Fig. 3a), with an average sensitivity 0.863 , specificity 0.763 and accuracy 0.841 . When applied to the internal validation, the average AUROC was 0.732 (Fig. 3b), with an average sensitivity of 0.773 , specificity of 0.763 and accuracy of 0.705 . When applied to the external validation, the AUROC dropped to 0.654 (Fig. 3c), with a sensitivity of 0.773 , specificity of 0.632 and accuracy of 0.641 (Table 2). Importantly, the average AUROC of the RM for HREV in the training was 0.983 (Fig. 3d), with an average sensitivity 0.948 , specificity 0.977 and accuracy 0.965. When applied to the internal validation, the average AUROC was 0.834 (Fig. 3e), sensitivity 0.916, specificity 0.969 and accuracy 0.947 . When applied to the external validation, the AUROC reached 0.736 (Fig. 3f), with sensitivity 0.690 , specificity 0.762 and accuracy 0.743 (Table 2 ).

Table 2

Performance of RMs for diagnosing of mild EV and HREV

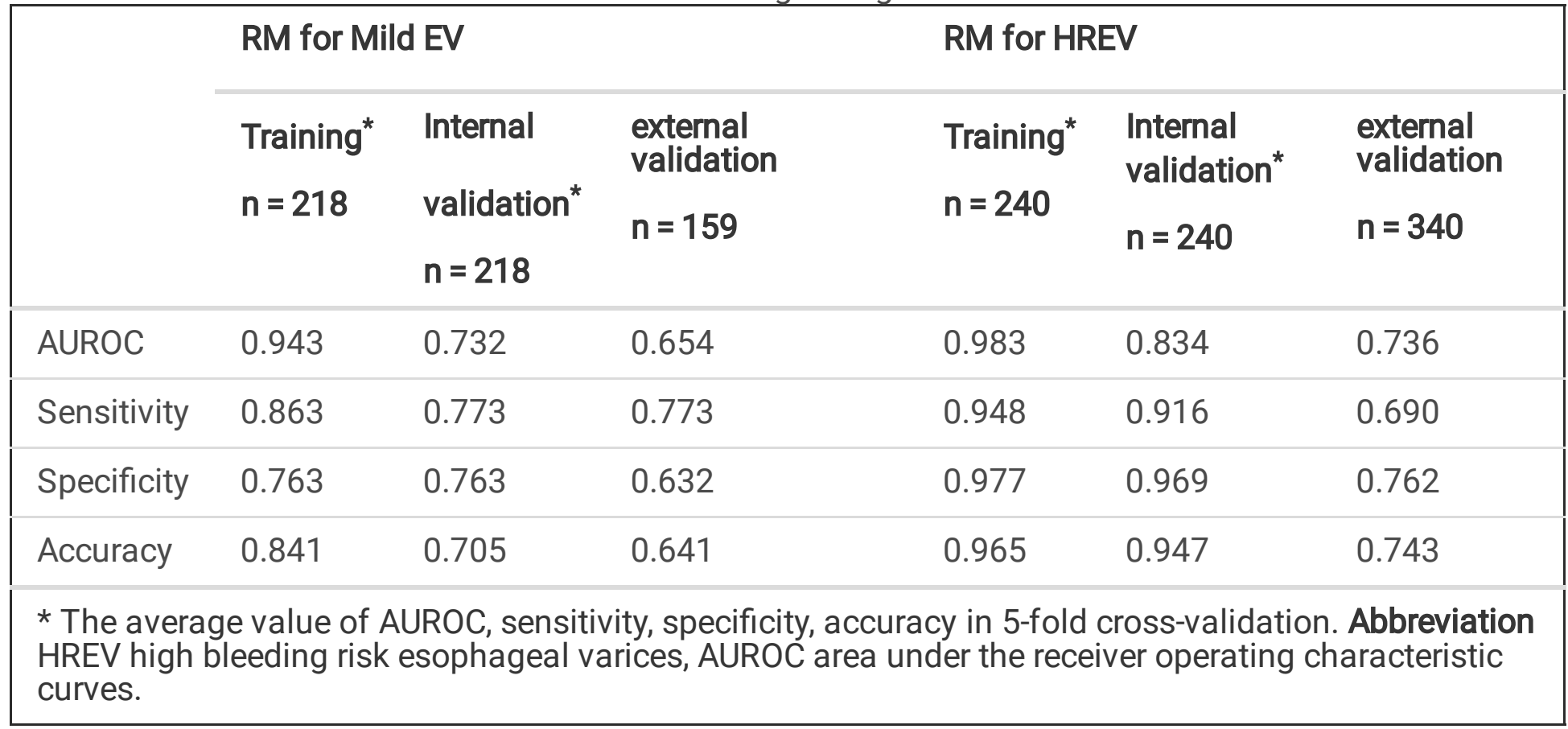

The calibration curve and DCA results of the RM were shown in Fig. 4a-f respectively. The DCA showed relatively good performance of the RM for diagnosing of mild EV and HREV. The net benefit was calculated by deducting the proportion of all patients who were false positive from the proportion who were true positive. Patients could benefit by using the proposed RM for diagnosing of HREV.

In external validation cohort, we also evaluated the efficacy of diagnosing of mild EV and HREV patients compared with Baveno VI and its expanded criteria. The accuracy, sensitivity and specificity were 0.629 , $0.813,0.149$ and $0.647,0.752,0.372$ of Baveno VI and expanded Baveno VI criteria, respectively. The NRI of RM were $49.0 \%$ and $32.8 \%$ compared with Baveno $\mathrm{VI}$ and expanded Baveno VI criteria respectivrly.

\section{Discussion}

Currently, doppler ultrasound, transient elastography, MRI and contrast-enhanced CT are all commonly used as non-invasive imaging methods for evaluation of portal hypertension in cirrhotic patients. Doppler 
ultrasonography is easy to operate and can show changes in velocity and direction of portal vein blood flow; But it is susceptible to intestinal gas, and the accuracy is affected by operator's experience.

Transient elastography is widely used for diagnosis of early liver cirrhosis by measuring the stiffness of the liver and spleen. However, it cannot be applied to patients with large ascites, and the accuracy is reduced in obese patients[19]. MRI and magnetic resonance elastography can also be applied to evaluate EV with high cost-effectiveness[20,21]. Interestingly, contrast-enhanced CT can be used to diagnose vascular diseases with high sensitivity and specificity[22]. liver, spleen, and portal vein system are clearly shown in the portal vein phase by three-dimensional(3D) reconstruction. Xie et al used CT to explore the risk of first EV bleeding in cirrhotic patients found that the total area of EV had significance for prediction bleeding[6]. The AUROC was 0.82 with cutoff value of $1.03 \mathrm{~cm}^{2}$. Han et al also used spleen hemodynamics (spleen iodine concentration and spleen volume) in dual-energy enhanced CT to predict EV in cirrhotic patients[7]. The AUROC for the detection of high-risk EV was 0.87 (95\% Cl: 0.77-0.94). However, There are no evidences of external validation above-mentioned studies.

Our study was to develop and validate the RM for identification of HREV in cirrhotic patients based on machine learning with contrast-enhanced CT imagine. Finally, 163 and 177 radiomic features were respectively included in mild EV and HREV RM. In the training, the average AUROC of RM for mild EV and HREV were 0.983 and 0.943 . As well, the average AUROC in the internal validation were 0.834 and 0.732 . It is suggested that RM were valuable in the diagnosing varices, especially identification of mild EV and HREV. Moreover, we enrolled 159 and 340 hepatitis B cirrhotic patients for external validation. The AUROC of RM for mild EV dropped to 0.654 . However, the AUROC of RM for HREV reached 0.736, which showed highly identification ability. Moreover, DCA and calibration curve exhibited good performance. It showed that RM has good value in screening HREV.

Baveno $\mathrm{VI}$ recommends that patients with a liver stiffness $<20 \mathrm{kPa}$ of transient elastography and PLT > $150 \times 10^{9} / \mathrm{L}$ have a very low risk of varices requiring treatment and can avoid endoscopy[16]. Sousa et al found this criteria had a high sensitivity but low specificity[23]. It suggested that, in clinical practice, there are still many patients had an unnecessary endoscopy. Salvador lauched expanded Baveno VI criteria for sparing more endoscopies[18]. But in our study, the expanded criteria with same problem of high sensitivity and low specificity as Baveno VI criteria were observed. The accuracy was not better than RM, yet. According to NRI, it exhibited the distinguishing efficiency of RM for diagnosing HREV had improved by $49.0 \%$ and $32.8 \%$ compared with Baveno $\mathrm{VI}$ and expanded Baveno $\mathrm{VI}$ criteria respectively. In conclusion, It showed that the value of the machine learning-based RM, as a novel non-invasive tool for diagnosing HREV in cirrhotic patients with highly accurate, surpassed previous non-invasive diagnostic tools.

Our study had the following limitations: First, the external validation cohort were only enrolled the cirrhotic patients with hepatitis $B$, and it was unable to determine the identification ability of HREV in cirrhotic patients with other etiologies. Second, in this study, a small samples and single center subjects were studied for external validation. Therefore, a larger and multi-center cohort for external validation are needed in the future.

Page $11 / 18$ 


\section{Abbreviations}

ALB albumin

ALT alanine aminotransferase

AST aspartate aminotransferase

AUROC under the receiver operating characteristic curves

Cr creatinine

CT computed tomography

DCA decision curve analysis

DCE dynamic contrast enhancement

EV esophageal varices

HOG histogram of oriented gradient

HREV high risk esophageal varices

HVPG Hepatic vein pressure gradient

INR international normalized ratio

NRI net reclassification improvement

PCA principal components analysis

PLT platelets counts

PT prothrombin time

PTA prothrombin time activity

RM radiomic model

ROI Regions of interest

SVM support vector machine

TB total bilirubin

\section{Declarations}


Funding This study was supported by the State Key Projects Specialized on Infectious Diseases, No. 2017ZX10203202-004, Digestive Medical Coordinated Development Center of Beijing Hospitals Authority, No. XXT24.

Availability of data and material The datasets used and analysed during the current study are available from the corresponding author.

Author contributions Dr.Yijie Yan,Yue Li and Chunlei Fan were contributed equally to collecting the data, development and validation RM. Huiguo Ding designed the project and was in charge of the manuscript. Zhenjia Ding performed the language polishing and scientific editing. Dr.Keqin Hu, Lei Li and Huiguo Ding were contributed equally to writing and editing. All authors read and approved the final manuscript.

\section{Conflict of interest statementᄆ}

All authors declare no conflict of interest.

\section{Ethics statements $\square$}

All procedures followed were in accordance with the ethical standards of the responsible committee on human experimentation (institutional and national) and with the Helsinki Declaration of 1975, as revised in 2008.

\section{References}

1. Roberts D, Best LM, Suzanne C, Freeman, et al. Treatment for bleeding oesophageal varices in people with decompensated liver cirrhosis: a network meta-analysis. Cochrane Database Syst Rev. 2021;2021:013155. doi:10.1002/2021/013155.Pub2 2021 Apr 10.

2. Ma J-L, He L-L, Li P, Jiang Yu, et al. Clinical features and Outcomes of Repeated Endoscopic Therapy for Esophagogastric Variceal Hemorrhage in Cirrhotic Patients: Ten-Year Real-World Analysis. Gastroenterol Res Pract. 2020;2020:5747563. doi:10.1155/2020/5747563. Epub 2020 May 15.

3. Tripathi D, Stanley AJ, Hayes PC, et al. U.K. Guidelines on the management of variceal haemorrhage in cirrhotic patients. Gut. 2015;64:1680-704. Apr 17, 2015.

4. Garcia-Tsao G, Abraldes JG, Berzigotti A, et al. Portal hypertensive bleeding in cirrhosis: risk stratification, diagnosis, and management: 2016 practice guidance by the American Association for the study of liver diseases. Hepatology. 2017;65:310-35. Jun 8, 2017.

5. Chinese Portal Hypertension Diagnosis and Monitoring Study Group (CHESS). Minimally Invasive Intervention Collaborative Group, Chinese Society of Gastroenterology, et al. Consensus on clinical application of hepatic venous pressure gradient in China (2018). Zhonghua Gan Zang Bing Za Zhi 2018; 26: 801-812, Nov 20, 2018.

6. Xie W, Chen FX, Zhu LY, et al. Risk assessment of first upper gastrointestinal bleeding using computerized tomoscanning in esophageal varices patients with cirrhosis and portal hypertension. 
Medicine (Baltimore) 2020; 99: e18923, Jan 5, 2020.

7. Han X, An W, Cao Q, et al. Noninvasive evaluation of esophageal varices in cirrhotic patients based on spleen hemodynamics: a dual-energy CT study. Eur Radiol. 2020;30:3210-6. Feb 18, 2020.

8. Inokuchi Y, Uematsu M, Takashina T. Diagnostic accuracy of the attenuation value in abdominal contrast enhanced dynamic multi-detector-row computed tomography for esophageal varices in patients with liver cirrhosis. Eur J Radiol Open. 2021;2021:100347. doi:10.1016/2021/100347. Ecollection 2021 Apr 23.

9. Ji GW, Zhu FP, Xu Q, et al. Radiomic features at contrast-enhanced CT predict recurrence in early stage hepatocellular carcinoma: a multi-institutional study. Radiology. 2020;294:568-79. Jan 14, 2020.

10. Chinese Society of Hepatology, Chinese Medical Association. Chinese guidelines on the management of liver cirrhosis. Zhonghua Gan Zang Bing Za Zhi. 2019;27:846-65. Nov 20, 2019.

11. Gamal Shiha A, Helmy A,et al. Asian-Pacific Association for the Study of the Liver (APASL) consensus guidelines on invasive and non-invasive assessment of hepatic fibrosis: a 2016 update. Hepatol Int. 2017;11(1):1-30. Jan 1, 2017.

12. David W, Crabb, Gene Y, Im G, Szabo,et al. Diagnosis and Treatment of Alcohol-Associated Liver Diseases: 2019 Practice Guidance From the American Association for the Study of Liver Diseases. Hepatology. 2020;71(1):306-33. Jan 1, 2020.

13. Cara L, Mack D, Adams DN, Assis, et al. Diagnosis and Management of Autoimmune Hepatitis in Adults and Children: 2019 Practice Guidance and Guidelines From the American Association for the Study of Liver Diseases. Hepatology. 2020;72(2):671-722. Aug 2, 2020.

14. Mohammed Eslam, Shiv K, Sarin VWai-Sun, Wong, et al. The Asian Pacific Association for the Study of the Liver clinical practice guidelines for the diagnosis and management of metabolic associated fatty liver disease. Hepatol Int. 2020; 14(6):889-919, Dec 6, 2020.

15. Chinese Society of Hepatology, Chinese Medical Association; Chinese Society of Gastroenterology, et al. Guidelines for the diagnosis and treatment of esophageal and gastric variceal bleeding in cirrhotic portal hypertension. Zhonghua Nei Ke Za Zhi 2016; 55:57-72, Feb 20, 2016.

16. De Franchis R. Expanding consensus in portal hypertension: report of the Baveno VI consensus workshop: stratifying risk and individualizing care for portal hypertension. J Hepatol. 2015;63:74352. Sep 3, 2015.

17. Kravetz D, Bildozola $M$, Argonz J, et al. Patients with ascites have higher variceal pressure and wall tension than patients without ascites. Am J Gastroenterol 2000; 95: 1770-1775, Jul 7, 2000.

18. Salvador Augustin M, Pons JB, Maurice, et al. Expanding the Baveno VI criteria for the screening of varices in patients with compensated advanced chronic liver disease. Hepatology. 2017;66(6):19808. Dec 6, 2017.

19. Ferraioli G, Wong VW, Castera L, et al. Liver Ultrasound Elastography: An update to the world federation for ultrasound in medicine and biology guidelines and recommendations. Ultrasound Med Biol. 2018;44:2419-40. Dec 12, 2018. 
20. Li H, Chen TW, Li ZL, et al. Albumin and magnetic resonance imaging-liver volume to identify hepatitis B-related cirrhosis and esophageal varices. World J Gastroenterol. 2015;21:988-96. Jan 3, 2015.

21. Jhang ZE, Wu KL, Chen $C B$, et al. Diagnostic value of spleen stiffness by magnetic resonance elastography for prediction of esophageal varices in cirrhotic patients. Abdom Radiol (NY). 2021;46(2):526-33. Feb 2, 2021.

22. Son JH, Lee SS, Lee Y, et al. Assessment of liver fibrosis severity using computed tomography-based liver and spleen volumetric indices in patients with chronic liver disease. Eur Radiol. 2020;30:348696. Jun 6, 2020.

23. Sousa M, Fernandes S, Proenca $L$, et al. The Baveno VI criteria for predicting esophageal varices: validation in real life practice. Rev Esp Enferm Dig. 2017;109:704-7. Oct 10, 2017.

\section{Figures}

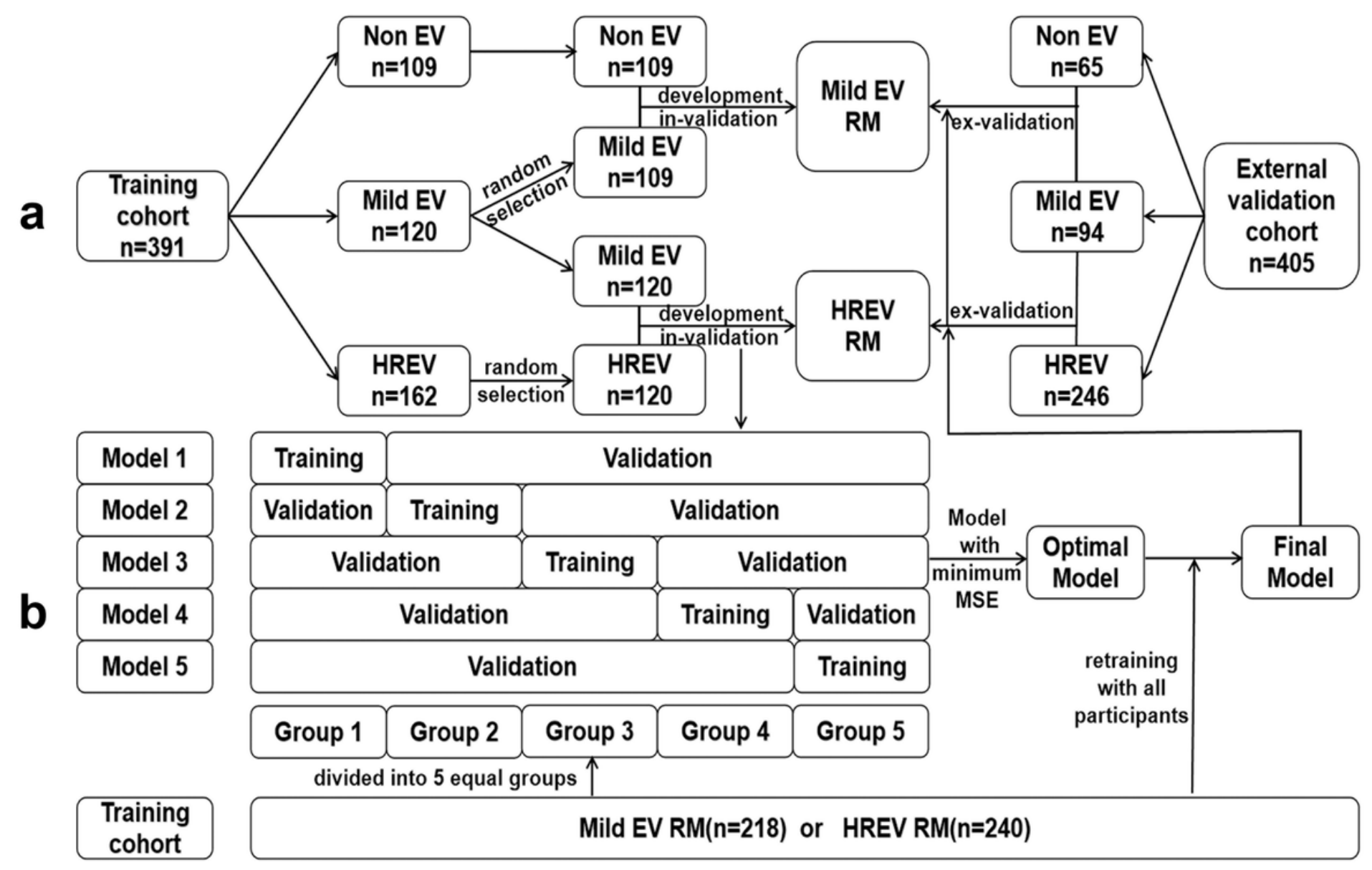

Figure 1 
The flowchart of participants enrollment and model establishment process. a: The flowchart of total 796 qualified participants were respectively enrolled in training and external validation cohort. b: The model sample source and process of 5 -fold cross validation. The participants were randomly divided into five groups, one group was used as training and development of radiomic model, and other 4 groups were used as internal validation.

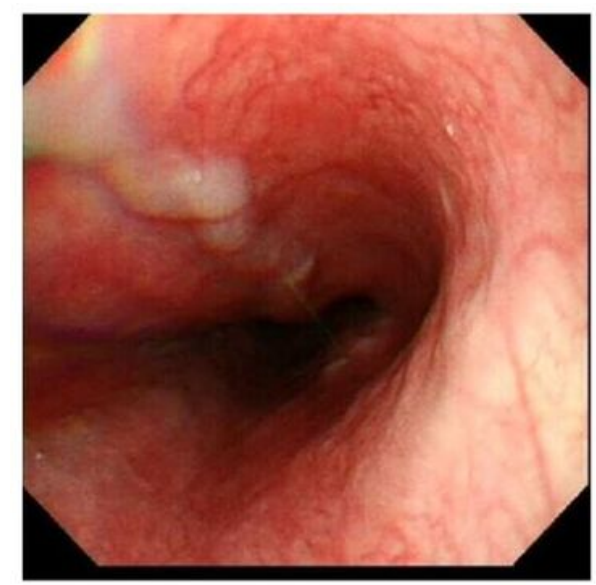

a

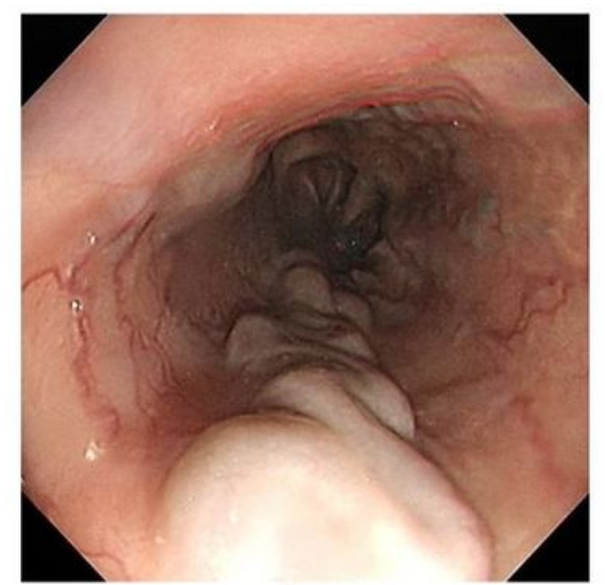

b

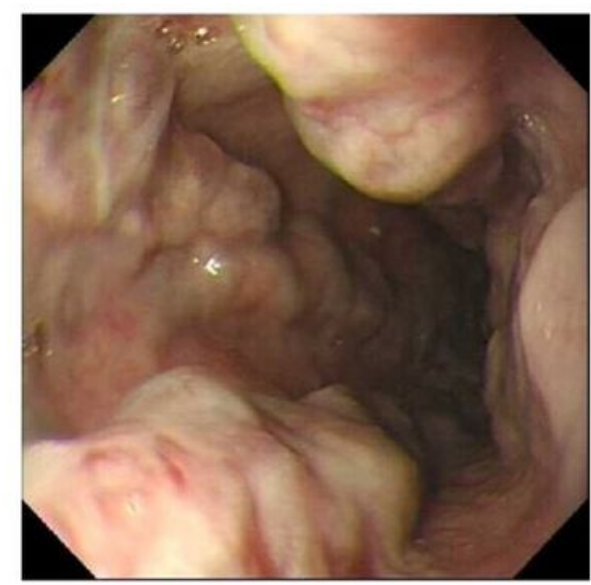

C

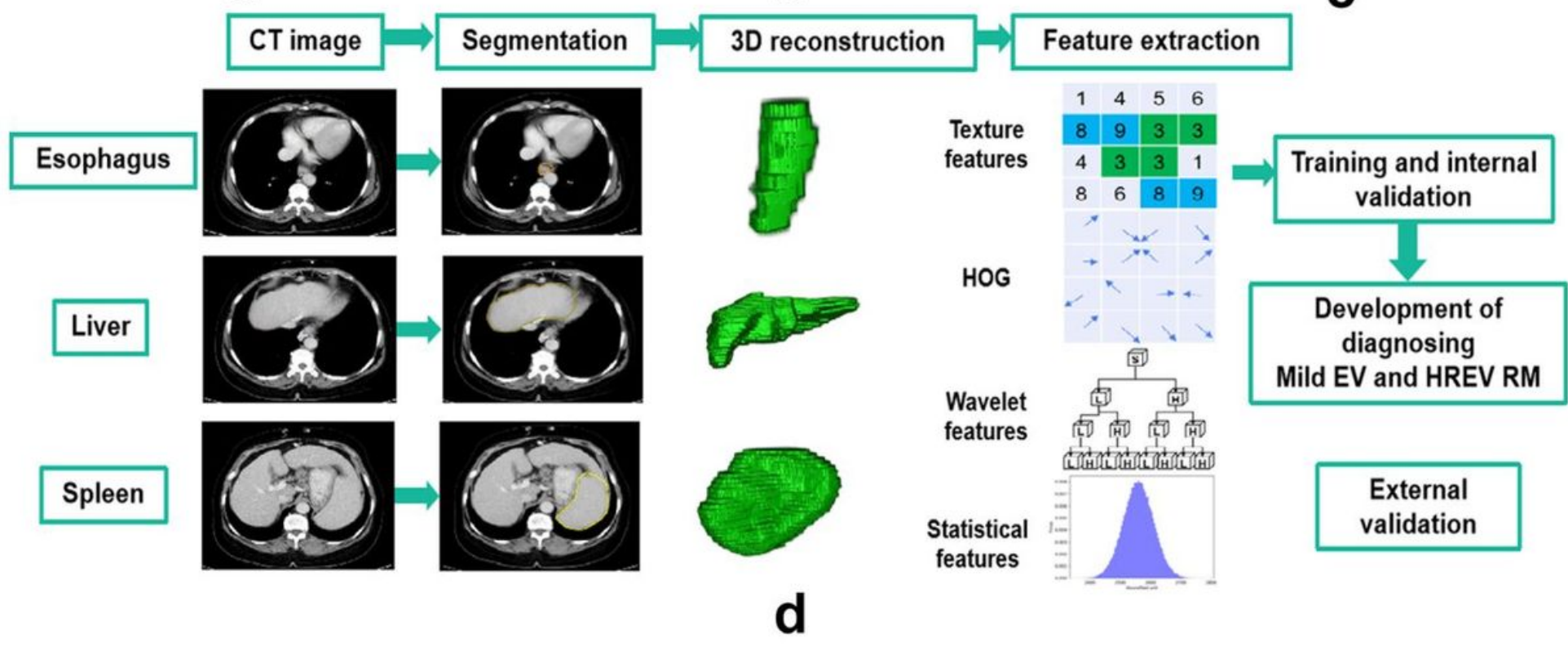

Figure 2

The general view of radiomic features extraction algorithm and endoscopic classification of the grade of $\mathrm{EV}$. The grade of EV by endoscopy: a: mild EV, b: moderate EV, and c: severe EV. $\mathrm{d}$ : The process of radiomic features extraction and $3 \mathrm{D}$ reconstruction. With manually segmented $\mathrm{ROI}$ on $\mathrm{CT}$ images of esophagus, liver, and spleen, were extracted. 

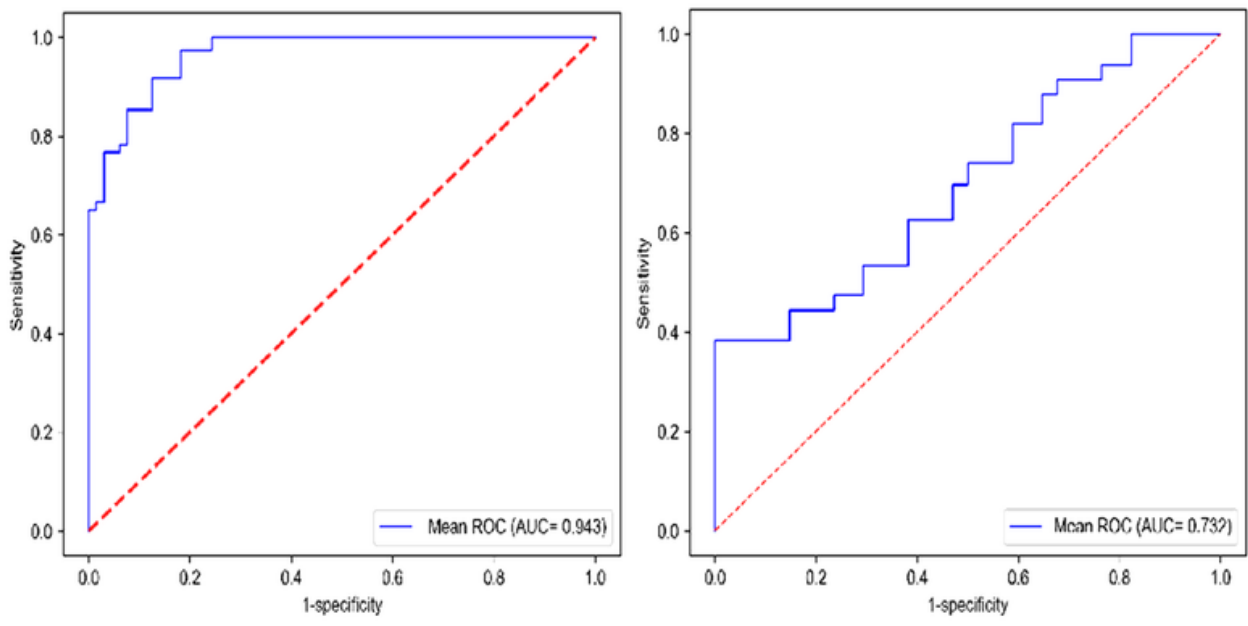

a

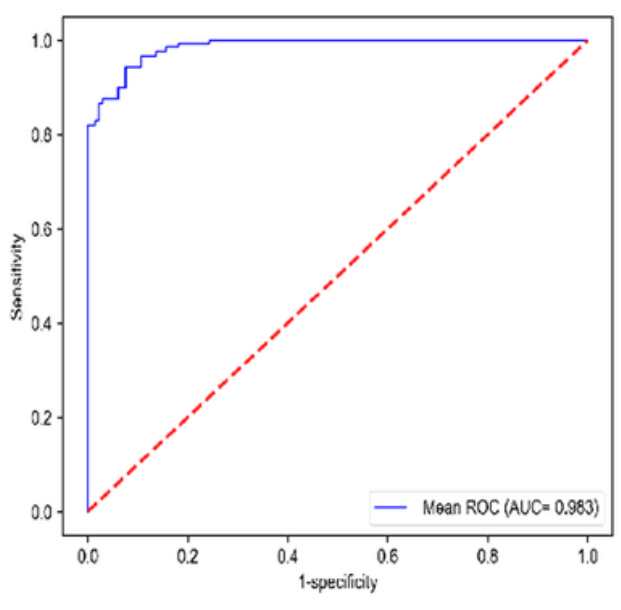

d b

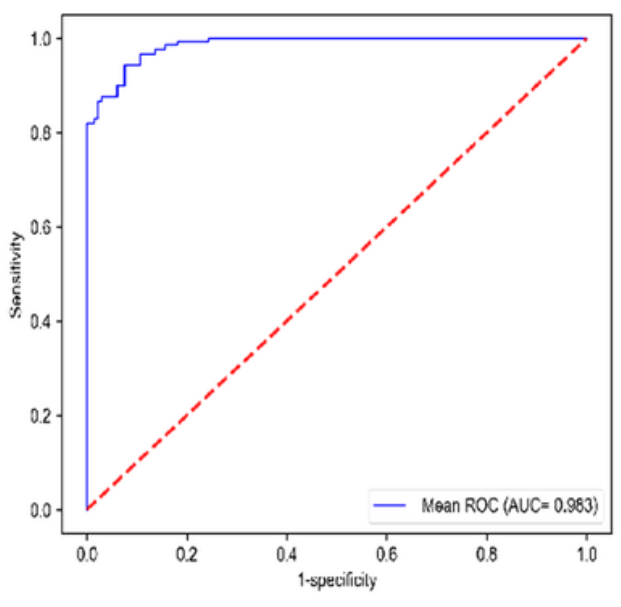

e

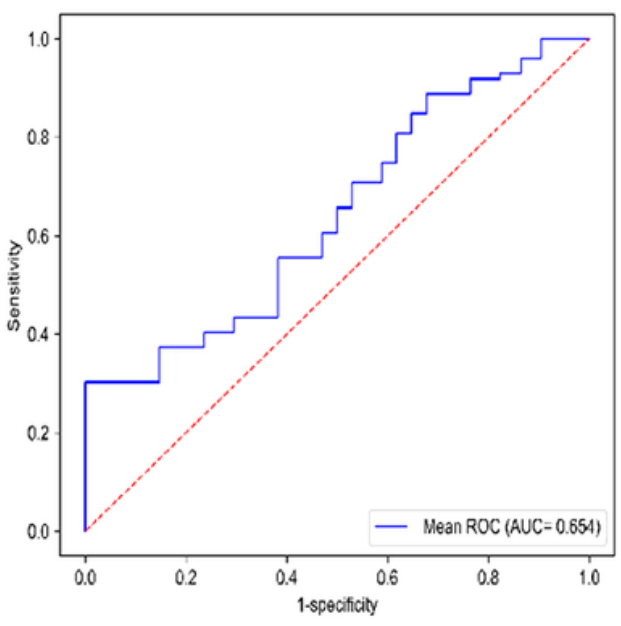

C

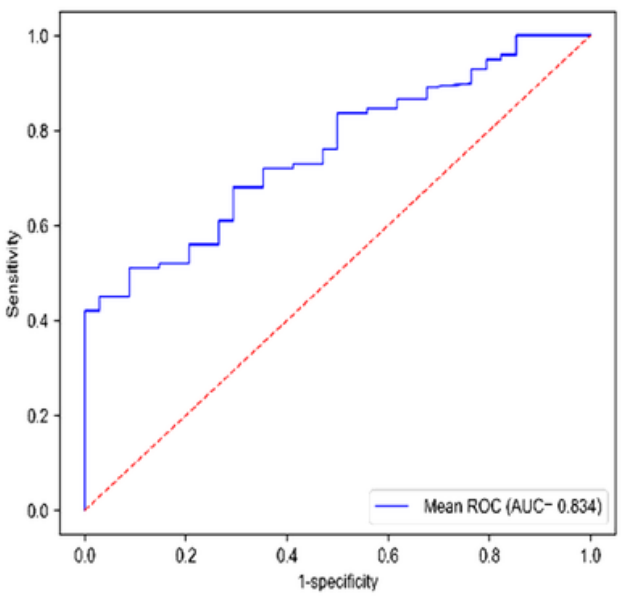

f

Figure 3

The average AUROC of the RMs for diagnosing of EV. The AUROC of the RM for diagnosing of mild EV in training (a), internal validation (b) and external validation (c) , and HREV in training (d), internal validation (e) and external validation (f). 

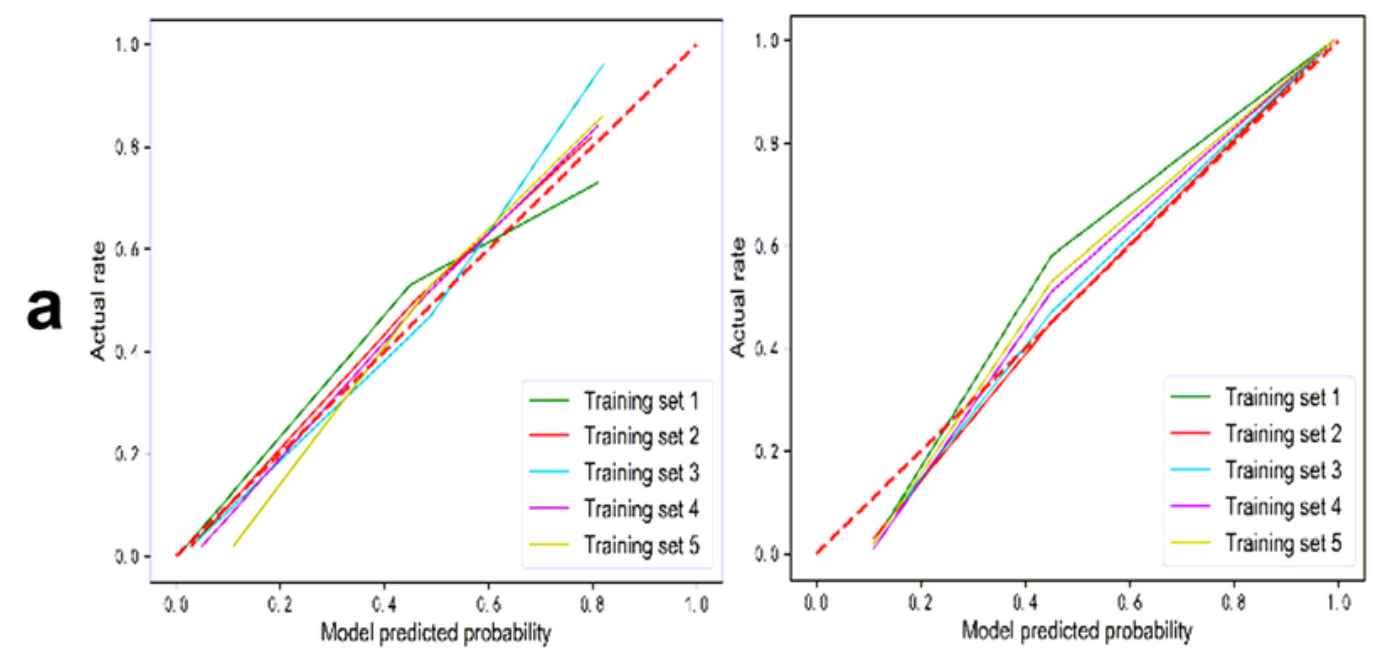

b
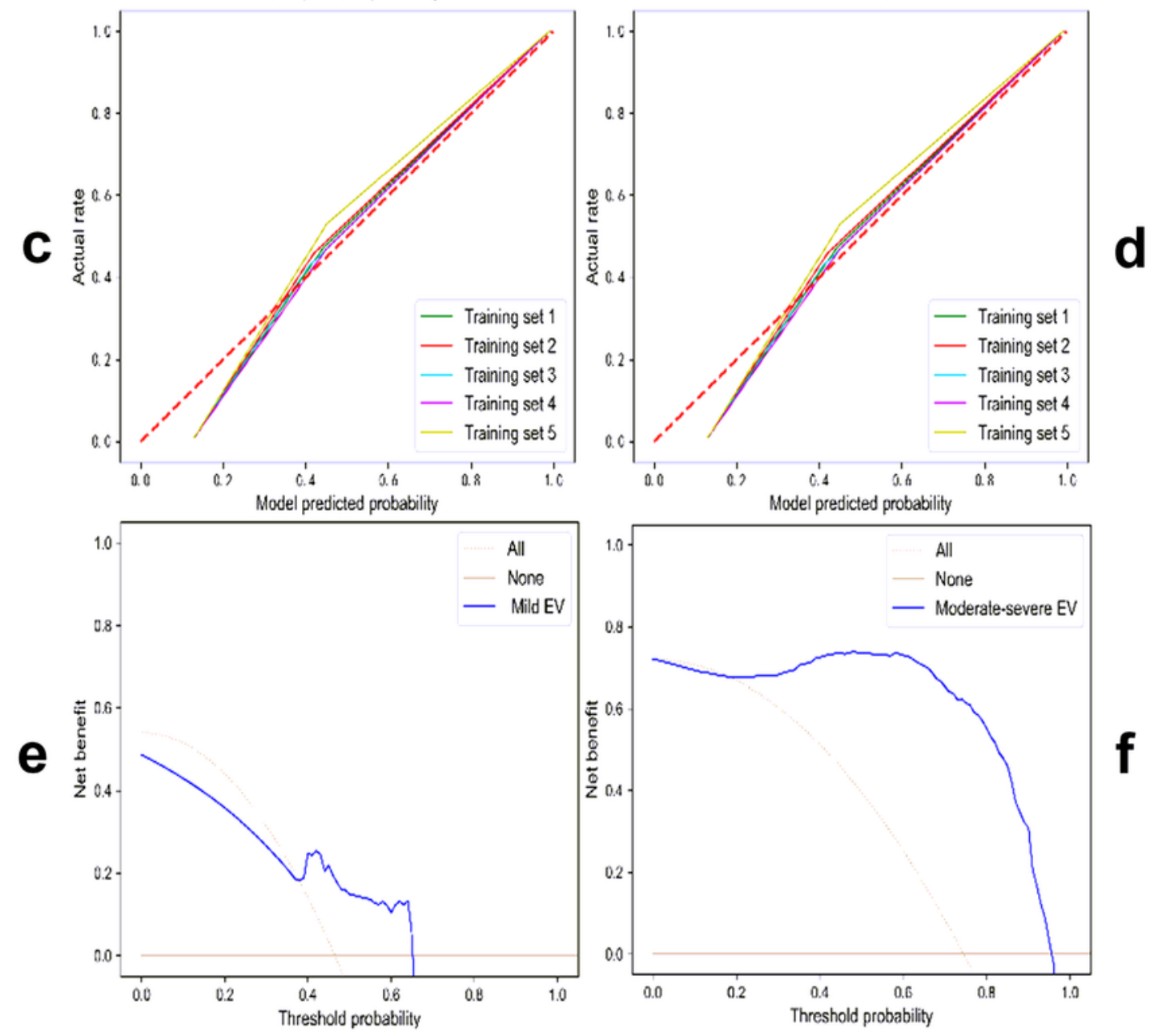

\section{Figure 4}

The calibration curve and DCA results of the RMs. Calibration curve of mild EV (a) and HREV (b) models in training cohort, and of mild EV (c) and HREV (d) in external validation cohort. The closer actual curve was to the fitting line(red dotted line), the more forecast result of RM were consistent with the actual situation. DCA for the RMs of mild EV (e) and HREV (f). when the blue line is farther away from the all line, it suggested the RM were benefit for clinical practices. 